\title{
The INGV tectonomagnetic network: 2004-2005 preliminary dataset analysis
}

\author{
F. Masci ${ }^{1}$, P. Palangio ${ }^{1}$, and A. Meloni ${ }^{2}$ \\ ${ }^{1}$ Istituto Nazionale di Geofisica e Vulcanologia, Osservatorio Geofisico di L'Aquila, Italy \\ ${ }^{2}$ Istituto Nazionale di Geofisica e Vulcanologia, Roma, Italy
}

Received: 21 June 2006 - Revised: 9 August 2006 - Accepted: 21 August 2006 - Published: 11 September 2006

\begin{abstract}
It is well established that earthquakes and volcanic eruption can produce small variations in the local geomagnetic field. The Italian Istituto Nazionale di Geofisica e Vulcanologia (INGV) tectonomagnetic network was installed in Central Italy since 1989 to investigate possible effects on the local geomagnetic field related to earthquakes occurrences. At the present time, total geomagnetic field intensity data are collected in four stations using proton precession magnetometers. We report the complete dataset for the period of years 2004-2005. The data of each station are differentiated respect to the data of the other stations in order to detect local field anomalies removing the contributions from the other sources, external and internal to the Earth. Unfortunately, no correlation between geomagnetic anomalies and the local seismic activity, recorded in Central Italy by the INGV Italian Seismic National Network, was found in this period. Some deceptive structures present in the differentiated data are pointed out.
\end{abstract}

\section{Introduction}

Some electromagnetic phenomena on the Earth surface and in the atmosphere can be associated with tectonic and volcanic activity in the Earth crust (Molchanov et al., 1995; Hayakawa and Fujinawa, 1994; Johnston, 1997). Volcanic eruptions and earthquakes can produce variations in the local geomagnetic field. The value of this variation is about few nT. Some studies report a variation about $1-5 \mathrm{nT}$ relating to earthquakes and a variation up to $10 \mathrm{nT}$ relating to volcanic activity (Del Negro and Currenti, 2003). In literature, two main phenomena have been suggested to explain the observed variation in local geomagnetic field: piezo-magnetic effects, resulting from variations of the rocks magnetization

Correspondence to: F. Masci

(masci@ingv.it) induced by mechanical or thermal stress and associated to slow variations (from weeks to months), and electro-kinetic effects, due to the presence of electric currents in the crust associated to rapid variations (from seconds to days) (Johnston, 1997; Johnston and Parrot, 1998; Hayakawa and Fujinawa, 1994). During the last two decades the INGV tectonomagnetic network has been intensively monitoring the total geomagnetic field in Central Italy in order to detect local anomalies related to the tectonic activity. At the beginning of the 1990s some anomalies in the geomagnetic field, probably related with the earthquakes occurrence, have been pointed out (Mele et al., 1994), but until now no evident correlation between tectonic activity and changes in the local magnetic field has been observed. In the last two years some inexplicable events in one station of the network have been occurred, as pointed out in Sect. 3, but no evident correlation with earthquakes occurrence has been found.

\section{The INGV Tectonomagnetic Network}

The Italian Istituto Nazionale di Geofisica e Vulcanologia tectonomagnetic network was installed in Central Italy since 1989 (Meloni et al., 1998) to investigate possible effects on the local geomagnetic field related to the tectonic activity. Total geomagnetic field intensity data has been detected in some stations using proton precession magnetometers. This network is part of the INGV L'Aquila Geomagnetic Observatory and it is located in Central Italy, in an area extending approximately between latitude $41.6^{\circ} \mathrm{N}$ and $42.8^{\circ} \mathrm{N}$, and between longitude $13.0^{\circ} \mathrm{E}$ and $14.3^{\circ} \mathrm{E}$. From a seismic point of view, Italy is one of the most active areas in Central Mediterranean with several active faults. General information on the geological structure of the Central Apennines are reported in Fig. 1 showing the known faults. For the details on the geological faults you can consult the List of the Active Faults and the Associate Earthquakes at

Published by Copernicus GmbH on behalf of the European Geosciences Union. 


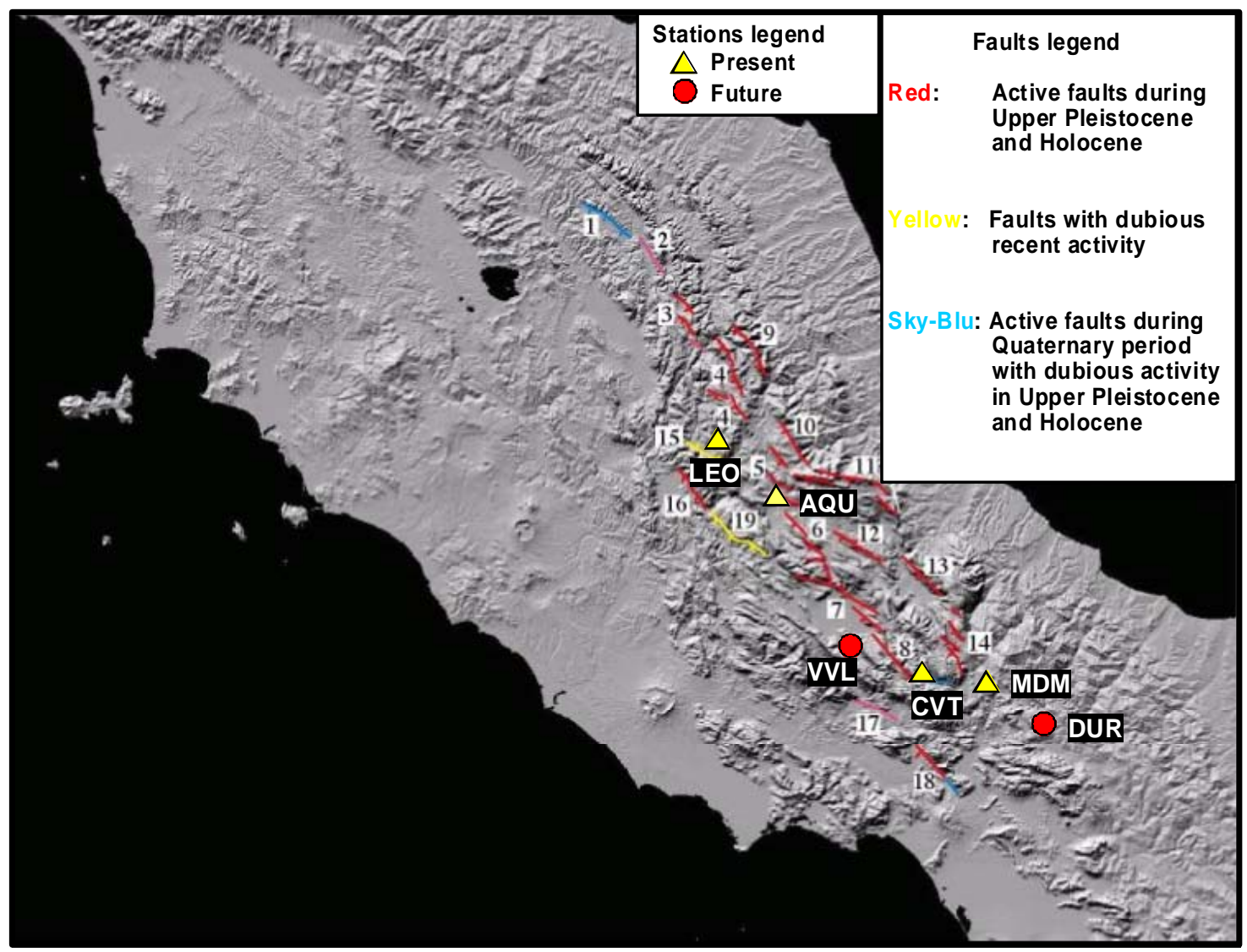

Fig. 1. Faults distribution in Central Apennines. The locations of the INGV tectonomagnetic network stations are also reported. (Adapted from the INGV-GNDT map of active faults in Central Italy).

the link http://emidius.mi.ingv.it/GNDT/P512/home.html of INGV-GNDT (Gruppo Nazionale per la Difesa dai Terremoti) site. In the Central Apennines changes in different parameters related to earthquakes have been described in literature. Bella et al. (1998) have described anomalous acoustic signals, anomalous electric and magnetic signals related to the $\mathrm{M}=3.9$ Gran Sasso earthquake occurred on 25 August 1992. Actually the INGV tectonomagnetic network consists in four stations located in L'Aquila (AQU), Monte di Mezzo (MDM), Civitella Alfedena (CVT) and Leonessa (LEO). The sampling rate of the stations is set to $15 \mathrm{~min}$ for each station except for AQU Intermagnet Station in which the sampling rate is $1 \mathrm{~min}$. The instrument accuracy is $0.1 \mathrm{nT}$ and the expected drift is $0.2 \mathrm{nT} / \mathrm{yr}$ (Meloni et al, 1998). In Fig. 1 are shown the locations of the network stations in Central Italy while in Table 1 is reported the list of the stations with their geographical coordinates. Two other stations, Duronia (DUR) and Villavallelonga (VVL) will be developed during 2006 and will be added to the tectonomagnetic network at the beginning of 2007 .

\section{$3 \quad 2004-2005$ dataset analysis}

Here we report the complete dataset of the INGV tectonomagnetic network for the period of two years 2004-2005 and the results of the preliminary analysis of the data. From the data analysis point of view, the total geomagnetic field data recorded in each station is differentiated respect to the data of the other stations in order to detect local field anomalies. The differentiation procedure removes the contributions from the other sources, external (i.e. electric currents in the ionosphere and magnetosphere) and internal to the Earth (i.e. secular trend of internal origin, due to the Earth's core electric currents). The only one remaining is due to local variation in crustal magnetization and to tectonic activity as well. Moreover, a daily mean of the differentiated data is calculated to remove the diurnal variation. In Fig. 2 are shown the daily mean of the differences among the stations of AQU, CVT and MDM. The differences with LEO station dataset are not reported because of the large number of gaps in the data due to technical and logistical problems. Bad weather conditions in winter, and the location of the LEO station in a rather inaccessible place, at relatively high altitude, also 
Table 1. List of the INGV tectonomagnetic network stations with the geographical coordinates.

\begin{tabular}{ll}
\hline \multicolumn{2}{c}{ Present stations } \\
\hline AQU & $42^{\circ} 23^{\prime} \mathrm{N} 13^{\circ} 19^{\prime} \mathrm{E} 682 \mathrm{~m}$ a.s.l. \\
CVT & $41^{\circ} 47^{\prime} \mathrm{N} 13^{\circ} 54^{\prime} \mathrm{E} 1020 \mathrm{~m}$ a.s.l. \\
MDM & $41^{\circ} 46^{\prime} \mathrm{N} 14^{\circ} 13^{\prime} \mathrm{E} 980 \mathrm{~m}$ a.s.l. \\
LEO & $42^{\circ} 33^{\prime} \mathrm{N} 13^{\circ} 04^{\prime} \mathrm{E} 1320 \mathrm{~m}$ a.s.l. \\
\hline \multicolumn{2}{c}{ Future stations } \\
\hline DUR & $41^{\circ} 39^{\prime} \mathrm{N} 14^{\circ} 27^{\prime} \mathrm{E} 910 \mathrm{~m}$ a.s.l. \\
VVL & $41^{\circ} 52^{\prime} \mathrm{N} 13^{\circ} 37^{\prime} \mathrm{E} 960 \mathrm{~m}$ a.s.l. \\
\hline
\end{tabular}

affect the continuity of the measurements. During the period of two years 2004-2005 no significant seismic activity has been registered in Central Italy (INGV Seismic Bulletin, 2004; INGV Seismic Bulletin, 2005). The maximum magnitude of the earthquakes registered during this period is about $\mathrm{M}=3$, so no significant anomalies in the local geomagnetic field is expected. In any case in Fig. 2 the differentiated data show some structures that can mislead. First of all, in the differentiated data can be pointed out some peaks with an amplitude about 2-3 nT in the AQU-MDM and in the AQUCVT differences. These peaks do not appear in the differentiated dataset MDM-CVT. For example, we can analyze the peak marked (a) in Fig. 2. In the lower panel of Fig. 3 is shown in details the peak (a) in the period of days JD=190 230 during 2004, whereas in the upper panel of Fig. 3 are reported the signals of the total geomagnetic field for the stations AQU e CVT in the same period. Note that the total geomagnetic field differences are reported as daily mean, while total geomagnetic field signals, shown in the upper panel, are reported with the original time interval of acquisition. Both the total geomagnetic fields show a magnetic storm beginning at JD=204 and ending at JD=211. In the same period of days a peak can be found in the differences between AQU and the two stations of MDM and CVT and it doesn't appear in the differences MDM-CVT. This effect can be explained with an evident latitude dependence of the magnetic storm between AQU and the other two stations. In the differences MDM-CVT the latitude dependence of the magnetic storm is not so obvious because the latitudes of the two stations are different about 1'. We can found similar peaks during 2004 on JD=24, 244 and 315, while during 2005 on JD=18, $128,135,150,191,237,243$ and 255. Analyzing the previous years dataset, the differentiated data show the (a)-type events every time a magnetic storm is occurred. Looking at the Fig. 2 is also obvious the presence of another kind of events marked as (b1) and (b2). In Fig. 4 are reported in details the two events, beginning on the JD=112 $2004 \mathrm{e}$ on the JD=38 2005. In the differences AQU-MDM both the events consist in a jump, between two levels, of $\sim 2.5 \mathrm{nT}$ dur-

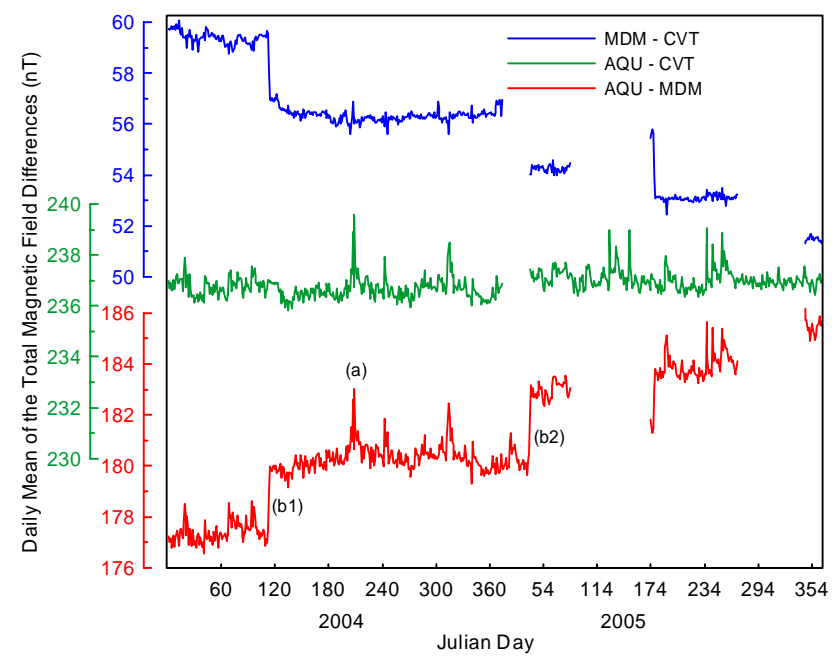

Fig. 2. Daily mean of the total geomagnetic field differences for the couple of station AQU-CVT, AQU-MDM, MDM-CVT during the period of two years 2004-2005. The colour of each plot is the same of the corresponding vertical axis.

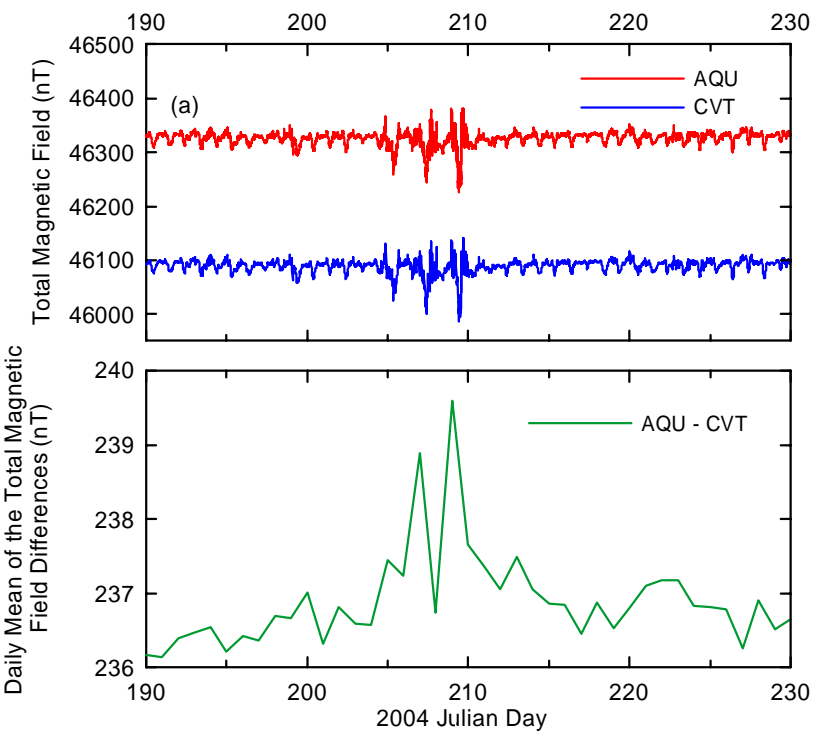

Fig. 3. The event marked (a) in Fig. 2 is reported in details for the AQU-CVT differences. In the upper panel are shown the total geomagnetic field intensities registered in the station of AQU and CVT for the period of days JD=190-230 2004. Both the signals show the presence of a magnetic storm beginning at JD=204 and ending at $\mathrm{JD}=211$. In the lower panel is reported the daily mean of the differences of the two signals shown in the upper panel. A clearly latitude dependence of the magnetic storm can be noted.

ing 2 days. These effects are due to the MDM total geomagnetic field intensity as they are present in the difference AQUMDM and MDM-CVT and are not evident in the differences AQU-CVT. We can exclude instrumental problems as, after the event (b1), we changed the MDM instrumentation with a 


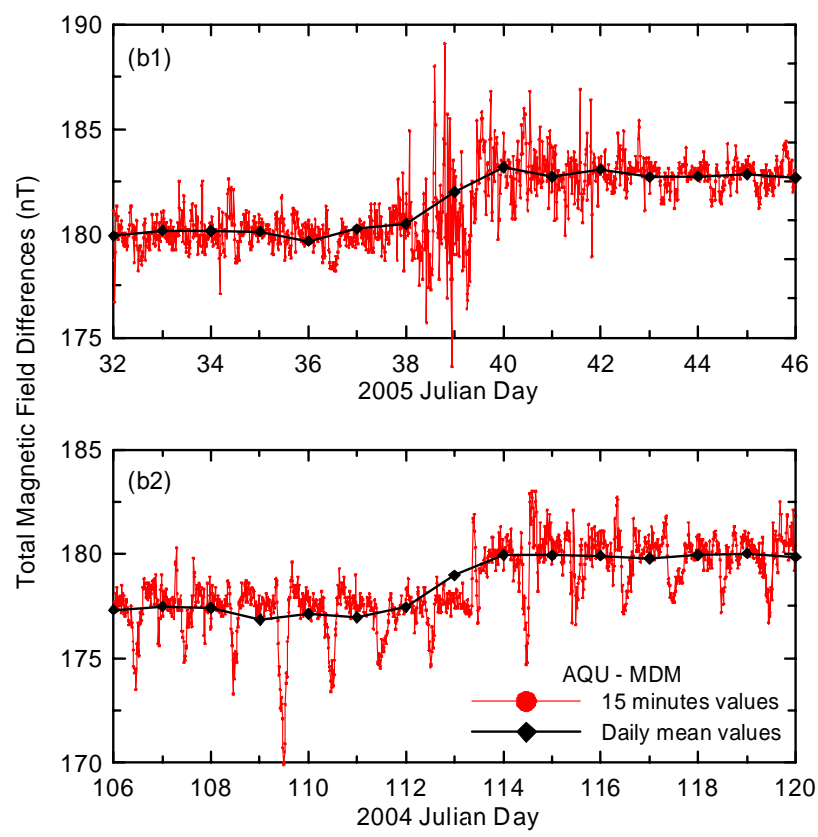

Fig. 4. The events marked (b1) and (b2) in Fig. 2 are reported in details. Both the events show a jump of $\sim 2.5 \mathrm{nT}$ in the differences AQU-MDM during 2 days. At the moment, there is no reasonable explanation for these events.

new calibrated magnetometer, but, after about a year we have recorded the event (b2) in MDM with the new instrumentation. Figure 2 shows a probable event like (b1) and (b2) for about the day JD=175 2005, happened unfortunately immediately after a gap in MDM dataset. Note that in MDM-CVT differences the mean values calculated in the periods of days $\mathrm{JD}=40-802005$ and $\mathrm{JD}=180-2702005$ are different probably happened also during the data gap occurred in the period of days $\mathrm{JD}=272-345$ since the mean values of the MDMCVT differences before JD=272 and after JD=345 are different about $1.5 \mathrm{nT}$. In the previous years the differentiated data involving the MDM station show two (b)-type events on 18 January 2003 and on 10 June 2003 (Di Mauro et al., 2006). Anyway, there are no relations between the (b)-type events occurred in the period of three years 2003-2005 and seismic activity in Central Italy. We can also exclude the relation of the (b)-type events with instrumental problems, as previously stressed, and with external influences because the sensor of MDM station is located in an area faraway from human activities. At this time, we have no reasonable explanation for these events and in the future it is needed more investigation to explain the cause. From the point of view of the data analysis, actually a new approach is attempted on the differentiated datasets using an autoregressive model to fit the data (Box and Jenkins, 1976). Preliminary analysis shows a second order autoregressive stationary model as the best fit. Future developments of this kind of analysis are in program trying to use the autoregressive model in a forecasting approach.

\section{Conclusions}

We have reported the whole dataset of the INGV tectonomagnetic network for the period of two years 2004-2005 as differences between the geomagnetic total field intensity collected in each network station. No relation with the local recorded earthquakes by the INGV Italian Seismic National Network has been found. On the other hand, during the period of two years 2004-2005 no significant seismic activity is registered in Central Italy. The maximum earthquakes magnitude registered during this period is about $\mathrm{M}=3$, so no significant variations in the local geomagnetic field is expected. Anyway, some evident structures that show latitude dependence of magnetic storms are highlighted in the differentiated data. Moreover, in the differences involving the MDM station dataset are shown some events with no reasonable explanation at this moment. More investigation is needed for a right interpretation of these events.

Acknowledgements. The authors thank the technical-administrative staff of the INGV L'Aquila Geomagnetic Observatory for the basic support in the research activity.

Edited by: P. F. Biagi

Reviewed by: one referee

\section{References}

Bella, F., Biagi, P. F., Caputo, M., Della Monica, G., Ermini, A., Plastino, W., and Sgrigna, V.: Anomalies in different parameters related to the M=3.9 Gran Sasso earthquake (1992), Phys. Chem. Earth, 23(9), 959-963, 1998.

Box, G. E. P. and Jenkins, G. M.: Time series analysis forecasting and control, Holden Day, San Francisco, USA, pp 575, 1976.

Del Negro, C. and Currenti, G.: Volcanomagnetic signals associated with the 2001 flank eruption of Mt. Etna (Italy), Geophys Res. Lett., 30(7), 1357-1361, 2003.

Hayakawa, M. and Fujinawa, Y. (Eds.): Electromagnetic Phenomena Related to Earthquake Prediction, Terra Sci. Pub. Comp., Tokyo, pp 677, 1994.

Di Mauro, D., Lepidi, S., Meloni, A., and Palangio, P.: Update on monitoring of magnetic and electromagnetic tectonic signals in Central Italy, Annals of Geophysics, in press, 2006.

INGV (Istituto Nazionale di Geofisica e Vulcanologia): Seismic Bullettin, Roma, 2004.

INGV (Istituto Nazionale di Geofisica e Vulcanologia): Seismic Bullettin, Roma, 2005.

Johnston, M. J. S.: Review of electrical and magnetic fields accompanying seismic and volcanic activity, Surv. Geophys., 18, 441-475, 1997.

Johnston, M. J. S. and Parrot, M.: Electromagnetic effects of earthquakes and volcanoes, Phys. Earth Planet. Int., Special Volume, 105, 109-295, 1998.

Mele, G., Meloni, A., and Palangio, P.: A tectonomagnetic effect detected in Central Italy , Ann. Geofis., 37(1), 17-25, 1994.

Meloni, A., Mele, G., and Palangio, P.: Tectonomagnetic field observation in central Italy 1989-1995, Phys. Earth Planet. Int., 105, 145-152, 1998. 
Molchanov, O. A., Hayakawa, M., and Rafalsky, V. A.: Penetration characteristics of electromagnetic emissions from an underground seismic source into the atmosphere, ionosphere and magnetosphere, J. Geophys. Res., 100, A2, 1691-1712, 1995. 